\title{
Article \\ A Double Logarithmic Transform Involving the Exponential and Polynomial Functions Expressed in Terms of the Hurwitz-Lerch Zeta Function
}

\author{
Robert Reynolds*(D) and Allan Stauffer (D) \\ Department of Mathematics and Statistics, York University, Toronto, ON M3J1P3, Canada; stauffer@yorku.ca \\ * Correspondence: milver@my.yorku.ca
}

\begin{abstract}
The object of this paper is to derive a double integral in terms of the Hurwitz-Lerch zeta function. Almost all Hurwitz-Lerch zeta functions have an asymmetrical zero-distribution. Special cases are evaluated in terms of fundamental constants. All the results in this work are new.
\end{abstract}

Keywords: Lerch function; double integral; Catalan's constant; Aprey's constant

check for updates

Citation: Reynolds, R.; Stauffer, A. A Double Logarithmic Transform Involving the Exponential and Polynomial Functions Expressed in Terms of the Hurwitz-Lerch Zeta Function. Symmetry 2021, 13, 1983. https://doi.org/10.3390/sym 13111983

Academic Editor:

Hari Mohan Srivastava

Received: 26 September 2021

Accepted: 18 October 2021

Published: 20 October 2021

Publisher's Note: MDPI stays neutral with regard to jurisdictional claims in published maps and institutional affiliations.

Copyright: (c) 2021 by the authors. Licensee MDPI, Basel, Switzerland. This article is an open access article distributed under the terms and conditions of the Creative Commons Attribution (CC BY) license (https:/ / creativecommons.org/licenses/by/ $4.0 /)$.

\section{Significance Statement}

P.S. Laplace (1749-1827) introduced the Laplace transform as part of their famous study of probability theory and celestial mechanics [1]. R.H Mellin (1854-1933) first gave a systematic formulation of the Mellin transformation and its inverse [2]. He used their transform to develop applications of the theory of special functions to the solution of hypergeometric differential equations.

The double Laplace transform is studied in the work of Debnath [3] to solve initial and boundary value problems in applied mathematics, and mathematical physics. The double Laplace transform has been used to study European vulnerable options under constant as well as stochastic (the Hull-White) interest rates [4].

In this work, we derive a double integral whose kernel involves generalized exponential, logarithmic and polynomial functions. The form of this kernel can be viewed as a double Laplace transform of logarithmic and polynomial functions when the powers of the variable $x$ in the exponential function is equal to one. On the other hand, since a polynomial is involved in this kernel, we can also consider this double integral as a double Mellin transform of the logarithm and exponential functions when $c=e=1$.

These integral types are also used to derive geometric probability constants by calculating the expected Euclidean distance $\delta(n)$ to the center of an $n$-dimensional cube [5], and have some similarities with the generalized hyperterminants in globally valid remainder terms for asymptotic expansions about saddles and contour endpoints of arbitrary order degeneracy derived from the method of steepest descents [6].

\section{Introduction}

In this paper, we derive the double integral given by

$$
\int_{0}^{\infty} \int_{0}^{\infty} t^{c m-1} x^{-e m+e-1} e^{-b t^{c}-d x^{e}} \log ^{k}\left(a t^{c} x^{-e}\right) d x d t
$$

where the parameters $k, a, b, d, m \in \mathbb{C}, \operatorname{Re}(c)>0, \operatorname{Re}(e)>0$. The derivations follow the method used by us in [7]. This method involves using a form of the generalized Cauchy's integral formula given by

$$
\frac{y^{k}}{\Gamma(k+1)}=\frac{1}{2 \pi i} \int_{C} \frac{e^{w y}}{w^{k+1}} d w
$$


where $C$ is in general an open contour in the complex plane, which has the same value at the end points of the contour. We then multiply both sides by a function of $x$ and $t$, then take a definite double integral of both sides. This yields a definite integral in terms of a contour integral. Then, we multiply both sides of Equation (2) by another function of $y$ and take the infinite sums of both sides such that the contour integral of both equations are the same.

\section{Definite Integral of the Contour Integral}

We use the method shown in [7]. The variable of integration in the contour integral is $\alpha=w+m$. The cut and contour are in the first quadrant of the complex $\alpha$-plane. The cut approaches the origin from the interior of the first quadrant and the contour goes round the origin with zero radius and is on opposite sides of the cut. Using a generalization of Cauchy's integral formula, we replace equations by replacing $y$ by $\log ^{k}\left(a t^{c} x^{-e}\right)$ and multiplying by $t^{c m-1} x^{e(-m)+e-1} e^{-b t^{c}-d x^{e}}$ then taking the definite integral with respect $x \in[0, \infty)$ and $t \in[0, \infty)$ to obtain

$$
\begin{aligned}
& \frac{1}{\Gamma(k+1)} \int_{0}^{\infty} \int_{0}^{\infty} t^{c m-1} x^{-e m+e-1} e^{-b t^{c}-d x^{e}} \log ^{k}\left(a t^{c} x^{-e}\right) d x d t \\
& \quad=\frac{1}{2 \pi i} \int_{0}^{\infty} \int_{0}^{\infty} \int_{C} a^{w} w^{-k-1} t^{c(m+w)-1} x^{e(-(m+w))+e-1} e^{-b t^{c}-d x^{e}} d w d x d t \\
& \quad=\frac{1}{2 \pi i} \int_{C} \int_{0}^{\infty} \int_{0}^{\infty} a^{w} w^{-k-1} t^{c(m+w)-1} x^{e(-(m+w))+e-1} e^{-b t^{c}-d x^{e}} d x d t d w \\
& \quad=\frac{1}{2 \pi i} \int_{C} \frac{\pi a^{w} w^{-k-1} b^{-m-w} d^{m+w-1} \csc (\pi(m+w))}{c e} d w
\end{aligned}
$$

from Equation (3.381.10) in [8], where $\operatorname{Re}(w+m)>0, \operatorname{Re}(c)>0, \operatorname{Re}(e)>0, \operatorname{Re}(b)>$ $0, \operatorname{Re}(d)>0, \operatorname{Re}(m)>0$ and using the reflection formula for the Gamma function (25.4.1) in [9]. We are able to switch the order of integration over $\alpha, x$ and $t$ using Fubini's theorem since the integrand is of bounded measure over the space $\mathbb{C} \times[0, \infty) \times[0, \infty)$.

\section{The Hurwitz-Lerch Zeta Function and Infinite Sum of the Contour Integral}

\subsection{The Hurwitz-Lerch Zeta Function}

The Hurwitz-Lerch zeta function, shown in Section (25.14) in [9-11] has a series representation given by

$$
\Phi(z, s, v)=\sum_{n=0}^{\infty}(v+n)^{-s} z^{n}
$$

where $|z|<1$, vs. $\neq 0,-1, .$. and is continued analytically by its integral representation given by

$$
\Phi(z, s, v)=\frac{1}{\Gamma(s)} \int_{0}^{\infty} \frac{t^{s-1} e^{-v t}}{1-z e^{-t}} d t=\frac{1}{\Gamma(s)} \int_{0}^{\infty} \frac{t^{s-1} e^{-(v-1) t}}{e^{t}-z} d t
$$

where $\operatorname{Re}(v)>0$, and either $|z| \leq 1, z \neq 1, \operatorname{Re}(s)>0$, or $z=1, \operatorname{Re}(s)>1$.

\subsection{Infinite Sum of the Contour Integral}

Using Equation (2) and replacing $y$ by $\log (a)-\log (b)+\log (d)+i \pi(2 y+1)$ then multiplying both sides by

$$
-\frac{2 i \pi b^{-m} d^{m-1} e^{i \pi m(2 y+1)}}{c e}
$$

then taking the infinite sum over $y \in[0, \infty)$ and simplifying in terms of the Hurwitz-Lerch zeta function to obtain 


$$
\begin{aligned}
-\frac{(2 i \pi)^{k+1} e^{i \pi m} b^{-m} d^{m-1} \Phi\left(e^{2 i m \pi},-k, \frac{-i \log (a)+i \log (b)-i \log (d)+\pi}{2 \pi}\right)}{c e \Gamma(k+1)} & \\
=-\frac{1}{2 \pi i} \sum_{y=0}^{\infty} \int_{C} \frac{1}{c e} 2 i \pi b^{-m} d^{m-1} w^{-k-1} \exp (w(\log (a)-\log (b)+\log (d)) & +i \pi(2 y+1)(m+w)) d w \\
=-\frac{1}{2 \pi i} \int_{C} \sum_{y=0}^{\infty} \frac{1}{c e} 2 i \pi b^{-m} d^{m-1} w^{-k-1} \exp (w(\log (a)-\log (b)+\log (d)) & +i \pi(2 y+1)(m+w)) d w \\
=\frac{1}{2 \pi i} \int_{C} \frac{\pi a^{w} w^{-k-1} b^{-m-w} d^{m+w-1} \csc (\pi(m+w))}{c e} d w &
\end{aligned}
$$

from Equation (1.232.3) in [8], where $\operatorname{Im}(w+m)>0$ in order for the sum to converge.

\section{Definite Integral in Terms of the Hurwitz-Lerch Zeta Function}

Theorem 1. For all $k, a, b, d, m \in \mathbb{C}, \operatorname{Re}(c)>0, \operatorname{Re}(e)>0$,

$$
\begin{aligned}
& \int_{0}^{\infty} \int_{0}^{\infty} t^{c m-1} x^{-e m+e-1} e^{-b t^{c}-d x^{e}} \log ^{k}\left(a t^{c} x^{-e}\right) d x d t \\
& =-\frac{1}{c e}(2 i \pi)^{k+1} e^{i \pi m} b^{-m} d^{m-1} \Phi\left(e^{2 i m \pi},-k, \frac{-i \log (a)+i \log (b)-i \log (d)+\pi}{2 \pi}\right)
\end{aligned}
$$

Proof. Observe the right-hand side of Equations (3) and (7) are equal so we may equate the left-hand sides and simplify the factorial to yield the stated result.

Lemma 1.

$$
\begin{aligned}
\int_{0}^{\infty} \int_{0}^{\infty} t^{\frac{c}{2}-1} x^{\frac{e}{2}-1} e^{-b t^{c}-d x^{e}} & \log ^{k}\left(-t^{c} x^{-e}\right) d x d t \\
=-\frac{1}{\sqrt{b} c \sqrt{d} e} i^{k+2}(2 \pi)^{k+1} & \left(2^{k} \zeta\left(-k, \frac{i \log (b)-i \log (d)+2 \pi}{4 \pi}\right)\right. \\
& \left.-2^{k} \zeta\left(-k, \frac{1}{2}\left(\frac{i \log (b)-i \log (d)+2 \pi}{2 \pi}+1\right)\right)\right)
\end{aligned}
$$

Proof. Use Equation (8) and set $m=1 / 2, a=-1$ and simplify using entry (4) in the table below (64:12:7) in [12].

\section{Special Cases}

In this section, we evaluate Equation (8) for various values of the parameters in terms of special functions and fundamental constants. In this section, we use the following functions and fundamental constants; Euler's constant $\gamma$, Catalan's constant $C$, Glaisher's constant $A$, Aprey's constant $\zeta(3)$, Hurwitz zeta function $\zeta(s, a)$, hypergeometric function ${ }_{2} F_{1}(a, b ; c ; z)$, Polylogarithm function $L i_{n}(z)$ and Riemann zeta function $\zeta(s)$.

\section{Example 1.}

$$
\int_{0}^{\infty} \int_{0}^{\infty} t^{c m-1} x^{-e m+e-1} e^{-b t^{c}-d x^{e}} d x d t=\frac{\pi b^{-m} d^{m-1} \csc (\pi m)}{c e}
$$

Proof. Use Equation (8) and set $k=0$ and simplify using entry (2) in the table below $(64: 12: 7)$ in [12]. 


\section{Example 2.}

$$
\begin{array}{r}
\int_{0}^{\infty} \int_{0}^{\infty} \frac{e^{-2\left(t^{2}+x^{3}\right)}\left(t^{2 p} x^{2-3 p}-t^{2 m} x^{2-3 m}\right)}{t \log \left(\frac{t^{2}}{x^{3}}\right)} d x d t \\
=\frac{1}{6}\left(\tanh ^{-1}\left(e^{i \pi m}\right)-\tanh ^{-1}\left(e^{i \pi p}\right)\right)
\end{array}
$$

Proof. Use Equation (8) and form a second equation by replacing $m \rightarrow p$ and taking their difference and setting $k=-1, a=1, b=c=d=2, e=3$ and simplifying using entry (3) in the table below $(64: 12: 7)$ in [12].

\section{Example 3.}

$$
\begin{aligned}
\int_{0}^{\infty} \int_{0}^{\infty} \sqrt{x} e^{-2 t^{2}-3 x^{3}} \log \left(\log \left(-\frac{t^{2}}{x^{3}}\right)\right) d x d t \\
=\frac{\pi}{12 \sqrt{6}}\left(4 \log \Gamma\left(-\frac{i \log \left(\frac{3}{2}\right)}{4 \pi}\right)-4 \log \Gamma\left(-\frac{1}{2}-\frac{i \log \left(\frac{3}{2}\right)}{4 \pi}\right)+3 i \pi+\log (16)\right. \\
\left.\quad+2 \log (\pi)+4 \log \left(\log \left(\frac{3}{2}\right)\right)+\log \left(\frac{1}{\left(\log \left(\frac{3}{2}\right)-2 i \pi\right)^{4}}\right)\right)
\end{aligned}
$$

Proof. Use Equation (9) and take the first partial derivative with respect to $k$ and set $k=0, b=c=2, d=e=3$ and simplify using Equation (25.11.18) in [9].

\section{Example 4.}

$$
\int_{0}^{\infty} \int_{0}^{\infty} \frac{\sqrt{x} e^{-5 x^{3}-i t^{2}}}{\log ^{2}\left(\frac{i t^{2}}{5 x^{3}}\right)} d x d t=\frac{(-1)^{3 / 4} C}{3 \sqrt{5} \pi}
$$

Proof. Use Equation (9) and set $k=-2, a=i / 5, b=i, c=2, d=5, e=3$ and simplify using Equation (3) in [13].

\section{Example 5.}

$$
\int_{0}^{\infty} \int_{0}^{\infty} \frac{t^{\frac{1}{6}(\sqrt{2}-6)} x^{\frac{1}{4}(\sqrt{2}-4)} e^{-t^{\frac{1}{\sqrt{2}}}-x^{\frac{1}{\sqrt{2}}}}\left(x^{\frac{1}{6 \sqrt{2}}}-t^{\frac{1}{6 \sqrt{2}}}\right)}{\log \left(t^{\frac{1}{\sqrt{2}}} x^{-\frac{1}{\sqrt{2}}}\right)} d x d t=-\log (3)
$$

Proof. Use Equation (8) and form a second equation by replacing $m \rightarrow p$ and taking their difference and setting $k=-1, a=1, b=1, d=1, m=1 / 2, p=1 / 3, c=1 / \sqrt{2}, e=1 / \sqrt{2}$ and simplify using Equation (7) in [14].

\section{Example 6.}

$$
\begin{aligned}
& \int_{0}^{\infty} \int_{0}^{\infty} \frac{e^{-t^{\frac{1}{\sqrt{2}}}-x^{\frac{1}{\sqrt{2}}} x^{-\frac{m+p-1}{\sqrt{2}}-1}\left(x^{\frac{m}{\sqrt{2}}} t^{\frac{p}{\sqrt{2}}}-t^{\frac{m}{\sqrt{2}}} x^{\frac{p}{\sqrt{2}}}\right)}}{t \log \left(t^{\frac{1}{\sqrt{2}}} x^{-\frac{1}{\sqrt{2}}}\right)} d x d t \\
& =2 \log \left(\cot \left(\frac{\pi m}{2}\right) \tan \left(\frac{\pi p}{2}\right)\right)
\end{aligned}
$$

Proof. Use Equation (8) and form a second equation by replacing $m \rightarrow p$ and taking their difference and setting $k=-1, a=1, b=1, d=1, c=1 / \sqrt{2}, e=1 / \sqrt{2}$ and simplifying using entry (1) in the table below (64:12:7) in [12]. 


\section{Example 7.}

$$
\int_{0}^{\infty} \int_{0}^{\infty} \frac{t^{\frac{1}{8}(\sqrt{2}-8)} x^{\frac{1}{8}(\sqrt{2}-8)} e^{-t^{\frac{1}{\sqrt{2}}}-x^{\frac{1}{\sqrt{2}}}}\left(t^{\frac{1}{2 \sqrt{2}}}-x^{\frac{1}{2 \sqrt{2}}}\right)}{\log \left(t^{\frac{1}{\sqrt{2}}} x^{-\frac{1}{\sqrt{2}}}\right)} d x d t=4 \sinh ^{-1}(1)
$$

Proof. Use Equation (15) and set $m=\frac{2 \cot ^{-1}(1+\sqrt{2})}{\pi}, p=\frac{2 \tan ^{-1}(1+\sqrt{2})}{\pi}$ and simplify. Note this is a double integral representation for the Universal Parabolic constant, $P$, given in [15]. This integral has similarities with the generalized hyperterminants in [6].

\section{Example 8.}

$$
\int_{0}^{\infty} \int_{0}^{\infty} \frac{x^{3 / 2} e^{-t^{2}-x^{5}}}{4 \log ^{2}\left(\frac{t^{2}}{x^{5}}\right)+\pi^{2}} d x d t=\frac{\pi+\log (3-2 \sqrt{2})}{20 \sqrt{2} \pi}
$$

and

$$
\int_{0}^{\infty} \int_{0}^{\infty} \frac{x^{3 / 2} e^{-t^{2}-x^{5}} \log \left(\frac{t^{2}}{x^{5}}\right)}{\log ^{2}\left(\frac{t^{2}}{x^{5}}\right)+\frac{\pi^{2}}{4}} d x d t=0
$$

Proof. Use Equation (8) and set $k=-1, a=i, c=2, e=5, b=1, d=1, m=1 / 2$ and simplify using entry (1) in table below (64:12:7) in [12]; then, rationalize the denominator and equate real and imaginary parts.

\section{Example 9.}

$$
\int_{0}^{\infty} \int_{0}^{\infty} \frac{\sqrt{t} x^{3 / 2} e^{-t^{3}-x^{5}}\left(\log ^{2}\left(\frac{t^{3}}{x^{5}}\right)-\pi^{2}\right)}{\left(\log ^{2}\left(\frac{t^{3}}{x^{5}}\right)+\pi^{2}\right)^{2}} d x d t=-\frac{\pi}{360}
$$

and

$$
\int_{0}^{\infty} \int_{0}^{\infty} \frac{\sqrt{t} x^{3 / 2} e^{-t^{3}-x^{5}} \log \left(\frac{t^{3}}{x^{5}}\right)}{\left(\log ^{2}\left(\frac{t^{3}}{x^{5}}\right)+\pi^{2}\right)^{2}} d x d t=0
$$

Proof. Use Equation (8) and set $k=-1, a=-1, c=3, e=5, b=1, d=1, m=1 / 2$ and simplify using entry (1) in table below (64:12:7) in [12]; then, rationalize the denominator and equate real and imaginary parts.

\section{Example 10.}

$$
\begin{gathered}
\int_{0}^{\infty} \int_{0}^{\infty} \sqrt{x} e^{-5 t^{2}-5 x^{3}} \log \left(-\frac{t^{2}}{x^{3}}\right) \log \left(\log \left(-\frac{t^{2}}{x^{3}}\right)\right) d x d t \\
=\frac{1}{30} i \pi^{2} \log (2 i \pi)-\frac{2}{15} i \pi^{2} \log \left(\frac{A^{3}}{\sqrt[3]{2} \sqrt[4]{e}}\right)
\end{gathered}
$$

Proof. Use Equation (8) and take the first partial derivative with respect to $k$ and set $m=1 / 2, k=1, b=d=5, a=-1, c=2, e=3$ and simplify using Equation (8) in [14].

\section{Example 11.}

$$
\int_{0}^{\infty} \int_{0}^{\infty} \sqrt{x} e^{-5 t^{2}-5 x^{3}} \log ^{2}\left(-\frac{t^{2}}{x^{3}}\right) \log \left(\log \left(-\frac{t^{2}}{x^{3}}\right)\right) d x d t=\frac{7 \pi \zeta(3)}{15}
$$

Proof. Use Equation (8) and take the first partial derivative with respect to $k$ and set $m=1 / 2, k=2, b=d=5, a=-1, c=2, e=3$ and simplify using Equation (9) in [14]. 


\section{Example 12.}

$$
\int_{0}^{\infty} \int_{0}^{\infty} \frac{\sqrt{t} e^{-t^{3}-x^{2}}}{\sqrt{\log \left(\frac{i t^{3}}{x^{2}}\right)}} d x d t=\frac{1}{6}(-1)^{3 / 4} \sqrt{\pi}\left(\zeta\left(\frac{1}{2}, \frac{7}{8}\right)-\zeta\left(\frac{1}{2}, \frac{3}{8}\right)\right)
$$

Proof. Use Equation (8) and set $m=1 / 2, k=-1 / 2, b=1, a=i, c=3, d=1, e=2$ and simplify using entry (4) in the table below (64:12:7) in [12].

\section{Example 13.}

$$
\begin{aligned}
& \int_{0}^{\infty} \int_{0}^{\infty} \sqrt{t} e^{-x^{2}+(-1-i) t^{3}} \log ^{\frac{i}{2}}\left(\frac{i t^{3}}{x^{2}}\right) d x d t \\
& \quad=\frac{1}{3}(-1)^{\frac{7}{8}+\frac{i}{4}} 2^{-\frac{1}{4}+i} \pi^{1+\frac{i}{2}}\left(\zeta\left(-\frac{i}{2}, \frac{13}{16}+\frac{i \log (2)}{8 \pi}\right)-\zeta\left(-\frac{i}{2}, \frac{5}{16}+\frac{i \log (2)}{8 \pi}\right)\right)
\end{aligned}
$$

Proof. Use Equation (8) and set $m=1 / 2, k=-i / 2, b=1+i, a=i, c=3, d=1, e=2$ and simplify using entry (4) in the table below (64:12:7) in [12].

\section{Example 14 .}

$$
\int_{0}^{\infty} \int_{0}^{\infty} \frac{t e^{-t^{3}-x^{2}}}{\sqrt[3]{x} \log \left(\frac{i t^{3}}{x^{2}}\right)} d x d t=-\frac{2}{9}\left(-\frac{1}{2}+\frac{i \sqrt{3}}{2}\right){ }_{2} F_{1}\left(\frac{3}{4}, 1 ; \frac{7}{4} ;-\frac{1}{2}-\frac{i \sqrt{3}}{2}\right)
$$

Proof. Use Equation (8) and set $m=2 / 3, k=-1, b=1, a=i, c=3, d=1, e=2$ and simplify using Equation (9.559) in [8].

\section{Example 15.}

$$
\begin{gathered}
\int_{0}^{\infty} \int_{0}^{\infty} t^{3 m-1} x^{1-2 m} e^{-d\left(t^{3}+x^{2}\right)} \log ^{k}\left(-\frac{t^{3}}{x^{2}}\right) d x d t= \\
\quad-\frac{i(2 i)^{k} \pi^{k+1} e^{-i \pi m} L i_{-k}\left(e^{2 i m \pi}\right)}{3 d}
\end{gathered}
$$

Proof. Use Equation (8) and set $b=d, a=-1, c=3, e=2$ and simplify using Equation (25.14.3) in [9].

\section{Example 16.}

$$
\int_{0}^{\infty} \int_{0}^{\infty} \sqrt{t} e^{-d\left(t^{3}+x^{2}\right)} \log ^{k}\left(-\frac{t^{3}}{x^{2}}\right) d x d t=-\frac{(2 i)^{k}\left(2^{k+1}-1\right) \pi^{k+1} \zeta(-k)}{3 d}
$$

Proof. Use Equation (26) and set $m=1 / 2$ and simplify using

\section{Example 17.}

$$
\int_{0}^{\infty} \int_{0}^{\infty} \frac{\sqrt{t} e^{-t^{3}-x^{2}}\left(\pi^{2}-3 \log ^{2}\left(\frac{t^{3}}{x^{2}}\right)\right)}{\left(\log ^{2}\left(\frac{t^{3}}{x^{2}}\right)+\pi^{2}\right)^{3}} d x d t=\frac{\zeta(3)}{32 \pi^{3}}
$$

and

$$
\int_{0}^{\infty} \int_{0}^{\infty} \frac{\sqrt{t} e^{-t^{3}-x^{2}} \log \left(\frac{t^{3}}{x^{2}}\right)\left(\log ^{2}\left(\frac{t^{3}}{x^{2}}\right)-3 \pi^{2}\right)}{\left(\log ^{2}\left(\frac{t^{3}}{x^{2}}\right)+\pi^{2}\right)^{3}} d x d t=0
$$

Proof. Use Equation (27) and set $k=-3, d=1$, rationalize the denominator and compare real and imaginary parts and simplify. 


\section{Example 18.}

$$
\int_{0}^{\infty} \int_{0}^{\infty} \frac{\sqrt{t} e^{-t^{3}-x^{2}}\left(5 \log ^{4}\left(\frac{t^{3}}{x^{2}}\right)-10 \pi^{2} \log ^{2}\left(\frac{t^{3}}{x^{2}}\right)+\pi^{4}\right)}{\left(\log ^{2}\left(\frac{t^{3}}{x^{2}}\right)+\pi^{2}\right)^{5}} d x d t=\frac{5 \zeta(5)}{512 \pi^{5}}
$$

and

$$
\int_{0}^{\infty} \int_{0}^{\infty} \frac{\sqrt{t} e^{-t^{3}-x^{2}} \log \left(\frac{t^{3}}{x^{2}}\right)\left(\log ^{4}\left(\frac{t^{3}}{x^{2}}\right)-10 \pi^{2} \log ^{2}\left(\frac{t^{3}}{x^{2}}\right)+5 \pi^{4}\right)}{\left(\log ^{2}\left(\frac{t^{3}}{x^{2}}\right)+\pi^{2}\right)^{5}} d x d t=0
$$

Proof. Use Equation (27) and set $k=-5, d=1$, rationalize the denominator and compare real and imaginary parts and simplify.

\section{Example 19.}

$$
\int_{0}^{\infty} \int_{0}^{\infty} \sqrt{t} e^{-t^{3}-x^{2}} \sqrt{\log \left(-\frac{t^{3}}{x^{2}}\right)} d x d t=\left(\frac{1}{12}+\frac{i}{12}\right)(2 \sqrt{2}-1) \sqrt{\pi} \zeta\left(\frac{3}{2}\right)
$$

Proof. Use Equation (27) and set $k=1 / 2, d=1$ and simplify.

\section{Example 20.}

$$
\begin{aligned}
& \int_{0}^{\infty} \int_{0}^{\infty} \frac{\sqrt{t} e^{-t^{3}}-x^{2} \log \left(\log \left(-\frac{t^{3}}{x^{2}}\right)\right)}{\log \left(-\frac{t^{3}}{x^{2}}\right)} d x d t= \\
& \frac{1}{12}(\pi \log (2)+i(\gamma \log (4)-\log (2)(\log (8)+2 \log (\pi))))
\end{aligned}
$$

Proof. Use Equation (27) to take the first partial derivative with respect to $k$ and apply l'Hopital's rule as $k \rightarrow-1$ and simplify using Equation (37) in [16].

\section{Discussion}

In this work, we derived a double integral in terms of the Hurwitz-Lerch zeta function. We then used this integral to derive special cases in terms of other special functions and fundamental constants. We were also able to derive double integral representations for geometric constants [15] and double integrals associated with with generalized hyperterminants in [6]. We will be using our method to derive more double integrals in terms of special functions. We checked our results numerically using Mathematica by Wolfram.

Author Contributions: Conceptualization, R.R.; funding acquisition, A.S.; supervision, A.S. All authors have read and agreed to the published version of the manuscript.

Funding: Natural Sciences and Engineering Research Council of Canada, Grant No. 504070.

Institutional Review Board Statement: Not applicable.

Informed Consent Statement: Not applicable.

Data Availability Statement: Not applicable.

Conflicts of Interest: The authors declare no conflict of interest.

\section{References}

1. Simon, P.; De Laplace, M. Théorie Analytique des Probabilités; Courcier: Paris, France, 1820.

2. Poularikas, A.D. The Transforms and Applications Handbook, 2nd ed.; CRC Press LLC: Boca Raton, FL, USA, 2000.

3. Debnath, L. The Double Laplace Transforms and Their Properties with Applications to Functional, Integral and Partial Differential Equations. Int. J. Appl. Comput. Math. 2016, 2, 223-241. [CrossRef]

4. Yoon, J.-H.; Kim, J.-H. The pricing of vulnerable options with double Mellin transforms. J. Math. Anal. Appl. 2015, 422, 838-857. [CrossRef] 
5. $\quad$ Finch, S.R. f Mathematical Constants; Cambridge University Press: Cambridge, UK, 2003.

6. Bennett, T.; Howls, C.J.; Nemes, G.; Daalhuis, A.B.O. Globally Exact Asymptotics for Integrals with Arbitrary Order Saddles. SIAM J. Math. Anal. 2018, 50, 2144-2177. [CrossRef]

7. Reynolds, R.; Stauffer, A. A Method for Evaluating Definite Integrals in Terms of Special Functions with Examples. Int. Math. Forum 2020, 15, 235-244. [CrossRef]

8. Gradshteyn, I.S.; Ryzhik, I.M. Tables of Integrals, Series and Products, 6th ed.; Academic Press: Cambridge, MA, USA, 2000.

9. Olver, F.W.J.; Lozier, D.W.; Boisvert, R.F.; Clark, C.W. (Eds.) NIST Digital Library of Mathematical Functions; U.S. Department of Commerce, National Institute of Standards and Technology: Washington, DC, USA; Cambridge University Press: Cambridge, UK, 2010; With 1 CD-ROM (Windows, Macintosh and UNIX). MR 2723248 (2012a:33001).

10. Srivastava, H.M. Some general families of the Hurwitz-Lerch Zeta functions and their applications: Recent developments and directions for further researches. Proc. Inst. Math. Mech. Nat. Acad. Sci. Azerbaijan 2019, 45, 234-269. [CrossRef]

11. Srivastava, H.M. The Zeta and Related Functions: Recent Developments. J. Adv. Eng. Comput. 2019, 3, 329-354. [CrossRef]

12. Oldham, K.B.; Myland, J.C.; Spanier, J. An Atlas of Functions: With Equator, the Atlas Function Calculator, 2nd ed.; Springer: New York, NY, USA, 2009.

13. Marichev, O.; Sondow, J.; Weisstein, E.W. Catalan's Constant, From MathWorld-A Wolfram Web Resource. Available online: https: / / mathworld.wolfram.com/CatalansConstant.html (accessed on 1 September 2021).

14. Weisstein, E.W. Lerch Transcendent, From MathWorld-A Wolfram Web Resource. Available online: https://mathworld.wolfram. com/LerchTranscendent.html (accessed on 1 September 2021).

15. Reese, S.; Sondow, J. Universal Parabolic Constant, From MathWorld-A Wolfram Web Resource, created by Eric W. Weisstein. Available online: https:/ / mathworld.wolfram.com/UniversalParabolicConstant.html (accessed on 1 September 2021).

16. Sondow, J.; Weisstein, E.W. Riemann Zeta Function, From MathWorld-A Wolfram Web Resource. Available online: https: //mathworld.wolfram.com/RiemannZetaFunction.html (accessed on 1 September 2021). 